\title{
Pregas cutâneas vs impedância bioelétrica na avaliação da composição corporal de atletas: uma revisão crítica
}

\section{Skinfold thickness versus bioelectrical impedance for the evaluation of body composition in athletes: a critical review}

1 Universidade de São Paulo, Faculdade de Medicina de Ribeirão Preto, Laboratório de Nutrição e Metabolismo, Ribeirão Preto, SP. Brasil.

Recebido em 18/12/07 Aprovado em 24/07/08
Resumo - A composição corporal é fator determinante no desempenho esportivo em diversas modalidades, sendo necessária a utilização de instrumentos seguros, práticos e válidos para determinar a composição corporal e identificar as modificações que ocorrem no decorrer da temporada de treinamento. Com isso, o objetivo do presente estudo foi, através de uma revisão crítica, comparar estudos que avaliaram as técnicas de pregas cutâneas e impedância bioelétrica para estimar a composição corporal de atletas. Foram revisados estudos publicados de 1990 a 2007, identificados "on line" através dos bancos de dados PubMed, SportDiscus e Scielo. Para qualificar os estudos, sete elementos críticos foram avaliados: número de participantes, nível dos atletas estudados, métodos estatísticos empregados, uso de diferentes equações, controle dos testes, controle da amostra e método de referência. Como ferramenta na avaliação da composição corporal de atletas, IB e PC apresentam muitas vantagens em relação a outros métodos, principalmente, quanto à facilidade de aplicação, por ser um método não invasivo de determinação e de preço razoavelmente barato. A exatidão e confiança de resultados obtidos por esses métodos são altamente dependentes das condições do teste, das equações selecionadas e das características do grupo a ser avaliado (modalidade desportiva, gênero, idade e nível competitivo dos atletas). A técnica de PC parece mais confiável quando comparada com IB para estimar a composição corporal em atletas, principalmente, por existirem equações específicas para diferentes modalidades e situações, e pela falta de sensibilidade do método de IB às pequenas mudanças na composição corporal que ocorrem durante a temporada de treinamento.

Palavras-chave: Composição corporal; Pregas cutâneas; Impedância elétrica; Atletas.

Abstract - Body composition is a determinant factor for performance in different sports modalities. Safe, practical and validated instruments are necessary for the measurement of body composition and for the identification of alterations that occur during the training period. A critical review was performed comparing studies that apply skinfold thickness (SKF) and bioelectrical impedance (BIA) for the estimation of body composition in athletes. Studies published between 1990 and 2007 available online in the Pubmed, SportDiscus and Scielo databases were reviewed. Seven critical points were evaluated to rate the studies: number of subjects, performance level of the athletes studied, statistical methods applied, number of equations tested, test control, sample control, and gold standard used to compare the data. SKF and BIA present advantages for the assessment of body composition in athletes compared to other methods, such as their easy application, non-invasiveness and relatively low cost. The accuracy and reliability of the results obtained with these methods depend on test conditions, equations selected for data analysis and characteristics of the particular group (sports modality, gender, age and performance level). SKF seems to be a better method to estimate body composition in athletes because of the availability of specific equations for different sports modalities and because of the low sensitivity of BIA in detecting minimal alterations that occur during the training period.

Key words: Body composition; Skinfold thickness; Electric impedance; Athletes. 


\section{INTRODUÇÃO}

A composição corporal é uma variável determinante em diversas modalidades esportivas: uma grande quantidade de massa muscular é necessária para aumentar a potência e a força dos movimentos (levantamento de peso, atletismo, natação); da mesma forma, uma baixa porcentagem de gordura corporal é importante em modalidades na qual o atleta deve suportar o seu peso corporal (ginástica artística), além das modalidades em que as categorias são divididas pelo peso corporal (judô, karatê, luta livre) ou aquelas em que a estética do atleta e a plástica dos movimentos são fundamentais (dança, fisiculturismo) $)^{1}$. Assim, De Lorenzo et al. ${ }^{2}$ afirmam ser necessário que técnicos e atletas tenham a disposição instrumentos seguros, facilmente administráveis, rápidos e válidos para determinar a composição corporal e identificar as modificações que ocorrem com a restrição calórica ou o treinamento desportivo no decorrer da temporada de treinamento. A avaliação apropriada da composição corporal permite estimar o peso corporal ideal para a competição, comparar atletas dentro do mesmo grupo e monitorar modificações nos componentes magro e gordo do corpo durante a temporada de treinamento ${ }^{1-3}$. A estimativa da composição corporal constitui, também, a primeira etapa na identificação de possíveis distúrbios alimentares e no planejamento nutricional ${ }^{4}$.

As técnicas de pregas cutâneas (PC) e de impedância bioelétrica (IB) são consideradas simples, de custo razoavelmente baixo e não invasivas para estimar a composição corporal e por esses motivos têm recebido importante atenção da literatura com relação a sua utilização em atletas ${ }^{2,5-11}$. No entanto, as vantagens da utilização dessas técnicas ainda não foram totalmente estabelecidas nessa população ${ }^{12,13}$.

As medidas de PC são utilizadas para estimar, indiretamente, a densidade corporal e a porcentagem de gordura em pessoas saudáveis ativas e em atletas $^{2}$ e grande número de equações têm sido desenvolvidas para esse fim ${ }^{3,7,14,15}$. Entretanto, esse método pode apresentar limitações, pois o avaliador deve ser treinado para desenvolver a técnica com acurácia e precisão, além de escolher a equação adequada para evitar erros metodológicos ${ }^{4}$.

A IB é outra técnica bastante estudada e utilizada para obtenção de informações sobre a composição corporal de atletas de diferentes modalidades esportivas ${ }^{1}$. Huygens et al. ${ }^{8}$ concluíram que a estimativa da composição corporal de atletas através da IB é extremamente confiável. No entanto, essa técnica é bastante dependente das condições do teste e da escolha da equação a ser utilizada para os cálculos de composição corporal. Autores têm relatado falta de sensibilidade em detectar modificações na massa magra e gordura corporal, por ser influenciada pelas mudanças bruscas na hidratação dos atletas decorrentes de variações no volume e intensidade de treinamento e na dieta, dentro da temporada de treinamento ${ }^{4}$. Segal ${ }^{1}$ relata que a confiabilidade da IB se restringe a um grupo específico de atletas ou para aferições individuais.

Stewart \& Hannan ${ }^{13}$ afirmam que as vantagens da utilização da PC e da IB para estimar a composição corporal de atletas não estão bem estabelecidas. A existência de diversas técnicas para estimar a composição corporal e a divergência de opiniões encontrada na literatura em relação à utilização das mesmas, torna evidente a necessidade de esclarecer qual das metodologias apresentadas representa a técnica mais adequada para avaliação dessa população. Além disso, o número pequeno de estudos que comparam técnicas mais simples com métodos de referência contribui para a divergência de resultados encontrados na literatura, tendo em vista que essas técnicas estão disponíveis somente em grandes centros de pesquisa. Assim, tornase evidente a necessidade de avaliar a literatura científica disponível sobre avaliação da composição corporal de atletas, para determinar qual técnica proporciona as melhores estimativas e auxiliar a atuação dos profissionais da área.

O objetivo do presente estudo foi, através de uma revisão crítica, comparar estudos que avaliaram as técnicas de pregas cutâneas e impedância bioelétrica para estimar a composição corporal de atletas.

\section{PROCEDIMENTOS METODOLÓGICOS}

Existem na literatura diversos estudos sobre composição corporal, envolvendo diferentes métodos e equações para sua determinação, desenvolvidos em várias populações e com inúmeras finalidades. Os artigos revisados incluem os mais relevantes estudos dos últimos 17 anos (de 1990 a 2007), identificados "on line" através dos bancos de dados PubMed, SportDicus e Scielo. Foram analisadas somente publicações em língua portuguesa e inglesa, usando as palavras-chave "body composition", "skinfold thickness" e "bioelectrical impedance" em combinação com "athletes". Artigos citados por outros estudos foram identificados, consultados nas bases de dados citadas acima e incluídos no 
presente estudo quando atendessem aos critérios de inclusão. Foram selecionados somente os estudos que comparavam as técnicas de pregas cutâneas e impedância bioelétrica ou ambos com técnicas de referência. Os estudos que tinham o objetivo apenas de caracterizar um grupo de atletas com relação à composição corporal, avaliar a composição durante da temporada e/ou relacionar com parâmetros de saúde e de performance não foram inseridos.

Para qualificar os estudos, sete elementos críticos foram identificados de acordo com os pontos mais relevantes: 1) número de participantes; 2) nível dos atletas estudados; 3 ) métodos estatísticos empregados; 4) uso de diferentes equações; 5) controle dos testes; 6) controle da amostra; 7) comparação com método de referência. Um sistema de pontuação foi criado (adaptado de Vitalla \& Newhouse) ${ }^{16}$ para avaliar o nível de evidência e objetividade de cada estudo com a possibilidade máxima de alcançar 12 pontos por estudo. Seguindo esse procedimento, os estudos foram agrupados segundo os aspectos positivos para o método de PC ou para IB.

\section{RESULTADOS}

Para seleção e caracterização, 67 estudos foram avaliados quanto aos critérios de inclusão, dentre os quais 12 foram selecionados. A Tabela 1 mostra as características dos 12 estudos incluídos na presente revisão. Foram destacados os pontos mais relevantes de cada estudo como população, objetivos, técnicas utilizadas, resultados e conclusões.

A Tabela 2 demonstra o sistema de pontuação proposto e a pontuação dos estudos revisados. Dentre os artigos incluídos, cinco deles apontam para a utilização da técnica de PC e apenas três para a técnica de IB como forma mais confiável de avaliar a composição corporal de atletas. Além disso, quatro estudos mostraram conclusões imparciais em relação à escolha da técnica mais apropriada para determinação da composição corporal em atletas.

Maior pontuação foi encontrada para os estudos que mostraram aspectos positivos para utilização da PC (40) comparado com IB (21). Os estudos com conclusões imparciais somaram 30 pontos. Dos 12 estudos citados, apenas três trabalharam com atletas amadores. Os estudos que mostraram aspectos positivos para utilização da PC foram desenvolvidos com maior controle metodológico e de amostragem, quando comparados aos que apresentaram aspectos positivos para utilização da IB. O mesmo não foi encontrado com relação à utilização de métodos estatísticos.
Cinco dos estudos não utilizaram um método de referência para validar PC e IB. Um dos estudos encontrados alcançou 11 pontos de um total de 12 possíveis, sendo este pertencente ao grupo que apontou vantagens para utilização do método de PC.

\section{DISCUSSÃO}

O sistema de pontuação (Tabela 2) requer grande atenção. Parte dessa discussão será voltada a esclarecer e comentar o sistema de pontuação proposto no presente estudo e os critérios adotados. Esses pontos críticos incluíram: nível dos atletas estudados, métodos estatísticos, utilização de diferentes equações, controle dos testes, presença de grupo controle ou validação cruzada e comparação com método de referência. A segunda parte da discussão será destinada aos resultados e seus pontos mais pertinentes.

O nível dos atletas estudados foi utilizado como critério para pontuação dos estudos, pois é nesse nível desportivo que estão as maiores lacunas no conhecimento sobre composição corporal. A grande variação individual de massa isenta de gordura, predominante em atletas de elite ${ }^{4}$, dificulta a validação de técnicas simples e rápidas. Saunders et al. ${ }^{19}$ encontraram, em atletas de resistência, variação individual muito grande em relação ao nível de treinamento do atleta e que tal variação pode interferir na predição da composição corporal por IB.

Em relação à metodologia estatística adotada em cada estudo, desde que Bland \& Altman $^{20}$ detectaram deficiências nos métodos de reprodutibilidade para medições repetidas e propuseram nova metodologia para determinar a concordância entre duas medidas, esses testes têm sido utilizados para validar instrumentos diferentes comparandoos com métodos consagrados (padrão ouro). Essas ferramentas apresentam vantagens na comparação de medidas e estimativas.

A validade tanto do método de IB quanto de PC são estritamente dependentes da equação a ser utilizada na avaliação ${ }^{8}$. Grande atenção deve ser dada à sua aplicação em grupos específicos de atletas que apresentem características diferentes de adiposidade. Além disso, outros fatores como a quantidade de massa muscular e densidade óssea aumentada, idade e estado de hidratação contribuem para a dificuldade em escolher ou desenvolver novas equações ${ }^{1}$.

O delineamento do estudo, ou seja, o controle de aspectos que possam interferir na avaliação da 
Tabela 1. Características dos estudos revisados. PH: Pesagem hidrostática; PC: Pregas cutâneas; IB: Impedância bioelétrica; DXA: absortometria com raios X de dupla energia; NIR: Espectrometria infra-vermelha.

\begin{tabular}{|c|c|c|c|c|c|}
\hline Referência & Participantes & $\begin{array}{l}\text { Técnicas } \\
\text { Utilizadas }\end{array}$ & $\begin{array}{l}\text { Objetivo do } \\
\text { Estudo }\end{array}$ & $\begin{array}{c}\text { Outros } \\
\text { Controles }\end{array}$ & Resultados e Conclusão \\
\hline Opplinger et al. ${ }^{5}$ & 57 lutadores & $\mathrm{PC}, \mathrm{IB}$ e $\mathrm{PH}$ & $\begin{array}{l}\text { Comparar técni- } \\
\text { cas, equações e } \\
\text { aparelhos de IB }\end{array}$ & $\begin{array}{l}\text { Foram feitas me- } \\
\text { didas repetidas } \\
\text { para validação } \\
\text { das técnicas }\end{array}$ & $\begin{array}{c}\text { PC e IB são validadas } \\
\text { somente quando utilizadas } \\
\text { equações específicas }\end{array}$ \\
\hline Webter \& Barr $^{12}$ & $\begin{array}{l}56 \text { ginastas } \\
\text { olímpicos e } \\
\text { patinadores de } \\
\text { velocidade }\end{array}$ & PC e IB & $\begin{array}{l}\text { Comparar dife- } \\
\text { rentes fórmulas } \\
\text { de IB e PC }\end{array}$ & & $\begin{array}{c}\text { A técnica de PC é adequa- } \\
\text { da com uso de equação } \\
\text { específica. }\end{array}$ \\
\hline De Lorenzo et al. ${ }^{2}$ & $\begin{array}{l}43 \text { atletas de } \\
\text { pólo aquático, } \\
\text { judô e karatê }\end{array}$ & PC, IB e DXA & $\begin{array}{c}\text { Comparar } \\
\text { técnicas e moda- } \\
\text { lidades }\end{array}$ & $\begin{array}{l}\text { Atletas de nível } \\
\text { internacional }\end{array}$ & $\begin{array}{c}\text { Nenhuma das técnicas é } \\
\text { válida. Há a necessidade de } \\
\text { mais estudos para determi- } \\
\text { nar equações especificas } \\
\text { para cada modalidade }\end{array}$ \\
\hline Stewart \& Hannan ${ }^{13}$ & $\begin{array}{l}82 \text { atletas ciclis- } \\
\text { mo, tênis, remo, } \\
\text { rúgbi, corrida, } \\
\text { musculação, tria- } \\
\text { tlo e fisiculturistas }\end{array}$ & PC, IB e DXA & $\begin{array}{l}\text { Comparar técni- } \\
\text { cas e equações }\end{array}$ & $\begin{array}{l}\text { Validação do } \\
\text { avaliador, técni- } \\
\text { cas e equações }\end{array}$ & $\begin{array}{c}\text { Melhor predição da gordura } \\
\text { corporal por PC com equa- } \\
\text { ção de } 4 \text { pregas }\end{array}$ \\
\hline Yannakoulia et al.7 & 42 dançarinas & PC, IB e DXA & $\begin{array}{l}\text { Comparar } \\
\text { técnicas e } \\
\text { validar equação } \\
\text { específica para } \\
\text { dançarinas }\end{array}$ & & $\begin{array}{c}\text { A nova equação estabele- } \\
\text { cida através da técnica de } \\
\text { IB é válida para estimar a } \\
\text { composição corporal de } \\
\text { dançarinas }\end{array}$ \\
\hline Huygens et al. ${ }^{8}$ & $\begin{array}{l}49 \text { fisiculturistas } \\
\text { e atletas de força }\end{array}$ & PC e IB & $\begin{array}{l}\text { Comparar técni- } \\
\text { cas e equações }\end{array}$ & & $\begin{array}{c}\text { IB não parece confiável } \\
\text { quando comparada com PC. } \\
\text { A soma das dobras é mais } \\
\text { confiável que as equações }\end{array}$ \\
\hline Andreoli et al. ${ }^{9}$ & $\begin{array}{l}10 \text { atletas de } \\
\text { pólo aquático }\end{array}$ & $\begin{array}{c}\text { PC, IB, PH e } \\
\text { DXA }\end{array}$ & $\begin{array}{l}\text { Comparar técni- } \\
\text { cas e formas de } \\
\text { aplicar a IB }\end{array}$ & $\begin{array}{l}\text { Diferentes } \\
\text { posições de } \\
\text { aplicação dos } \\
\text { eletrodos }\end{array}$ & $\begin{array}{l}\text { IB e PC superestimam os } \\
\text { resultados de referência de- } \\
\text { terminados por } \mathrm{PH} \text { e DXA }\end{array}$ \\
\hline Utter et al. ${ }^{10}$ & $\begin{array}{l}129 \text { atletas esco- } \\
\text { lares wrestiling }\end{array}$ & $\mathrm{PC}, \mathrm{IB}$ e $\mathrm{PH}$ & $\begin{array}{l}\text { Comparar } \\
\text { técnicas com IB } \\
\text { perna-a-perna }\end{array}$ & $\begin{array}{l}\text { Controle da } \\
\text { ingestão ali- } \\
\text { mentar ,álcool, } \\
\text { diuréticos, } \\
\text { treinamento, } \\
\text { urina e estado } \\
\text { de hidratação }\end{array}$ & $\begin{array}{l}\text { PC é mais preciso que IB } \\
\text { perna-a-perna e deve ser } \\
\text { preferencialmente utilizado } \\
\text { para estimar gordura corpo- } \\
\text { ral em wrestlers }\end{array}$ \\
\hline Hetzler et al. ${ }^{18}$ & $\begin{array}{l}208 \text { atletas } \\
\text { escolares de } \\
\text { wrestling }\end{array}$ & PC e IB & $\begin{array}{l}\text { Comparar técni- } \\
\text { cas e equações }\end{array}$ & $\begin{array}{l}\text { Estado de hidra- } \\
\text { tação e diferen- } \\
\text { tes equações }\end{array}$ & $\begin{array}{l}\text { Os resultados obtidos pelas } \\
\text { técnicas de IB e PC mostra- } \\
\text { ram resultados diferentes } \\
\text { e alta variabilidade dos } \\
\text { mesmos dependendo da } \\
\text { equação utilizada }\end{array}$ \\
\hline
\end{tabular}


Tabela 2. Sistema de pontuação dos estudos e pontuação.

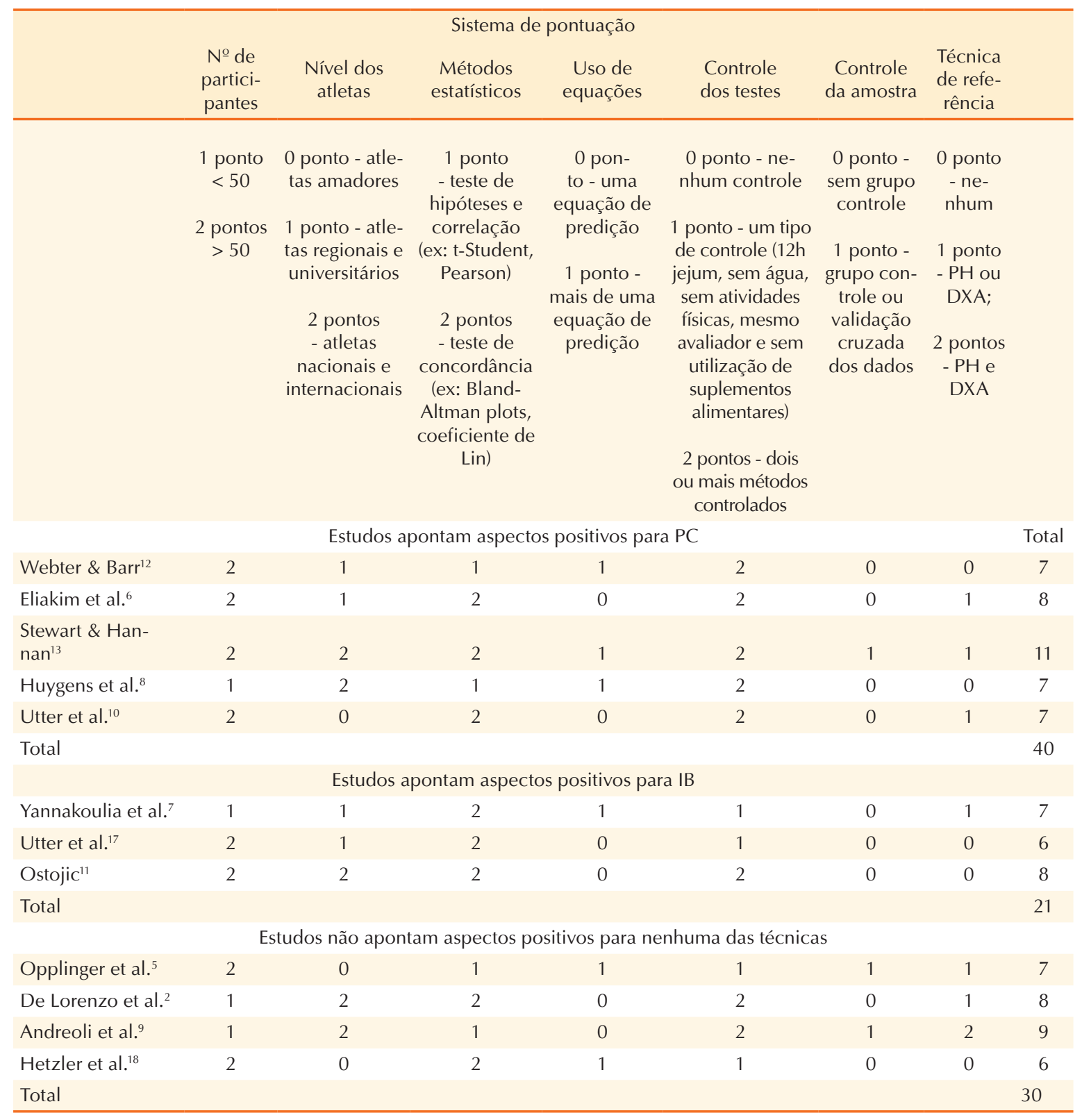

composição corporal foi outro importante critério de pontuação. Estudos comparativos mostram razoável grau de confiança ao método de IB apenas quando as condições do teste são bem controladas. Lukaski et al. ${ }^{21}$ em estudo comparando os métodos de IB e PH em condições controladas e não controladas, encontraram diferenças significativas na composição corporal de 104 atletas, utilizando diferentes métodos e em diferentes situações, com um erro de aproximadamente 30\% quando as condições do teste não eram controladas. $\mathrm{O}$ estado de hidratação parece ser a variável mais importante quando se utiliza IB ${ }^{1}$. Para a técnica de pregas cutâneas, a experiência do avaliador pode influenciar a confiabilidade do método ${ }^{6}$. Além disso, a maioria dos atletas de alto nível, principalmente, competidores de força, explosão muscular e fisiculturistas, utilizam algum tipo de suplemento alimentar durante a fase de treinamento que pode alterar a massa isenta de gordura, entretanto, seus efeitos na resistência, reatância e hidratação não são totalmente esclarecidos ${ }^{8}$. Na presente revisão, apenas três dos 12 estudos selecionados utilizaram grupo controle ou método de validação cruzada. Esses cuidados são de extrema importância para a confiança nos resultados.

Entre as décadas de 70 e 80, o modelo de dois compartimentos (massa gorda e massa isenta de 
gordura) era considerado o "padrão ouro" para estimar a composição corporal ${ }^{13}$. A PH é a técnica mais utilizada e estudada para estimar a densidade corporal, e utilizada por muitos autores como método referência até os dias atuais ${ }^{4}$. Entretanto, estudos recentes têm utilizado a técnica de absortometria com raios X de dupla energia para determinar a composição corporal ${ }^{22}$. Considerado o método "padrão ouro" para determinação da densidade mineral óssea $^{13}$, a DXA pode ser considerada um método de referência para estimar a composição corporal pelo crescente número de evidências encontradas na literatura recente $\mathrm{1}^{13,21,23}$.

Em relação aos resultados encontrados, os cinco estudos que mostraram aspectos positivos com relação ao método de pregas cutâneas somaram 40 pontos, contra 21 pontos somados pelos três estudos que observaram vantagens no uso de impedância bioelétrica e os 30 pontos dos três estudos que não apontaram aspectos positivos para nenhum dos métodos. Os resultados encontrados parecem apontar a técnica de PC como o melhor método, comparado com IB, para estimar a composição corporal em atletas. Analisando as pontuações dos artigos, observa-se que os trabalhos que apontaram as pregas cutâneas como técnica de escolha estudaram, em sua maioria, atletas de alto nível; apresentaram desenho experimental mais controlado, em relação aos testes; compararam com técnicas de referência e analisaram com ferramentas estatísticas apropriadas.

As mensurações das pregas cutâneas proporcionam informações confiáveis acerca da gordura corporal e de sua distribuição ${ }^{4}$. Existem duas formas de utilização das PC. A primeira soma os escores de pregas cutâneas para indicar a adiposidade dos indivíduos. A soma das pregas cutâneas e os valores individuais refletem modificações absolutas ou percentuais da gordura corporal antes e após um programa de intervenção ${ }^{12}$. Huygens et al. ${ }^{8}$ sugerem que a soma das pregas cutâneas é uma alternativa bastante válida para indicar a gordura subcutânea e o desenvolvimento muscular de fisiculturistas. Uma segunda forma de utilização das pregas cutâneas incorpora as equações matemáticas específicas para determinadas populações e que permitem prever a densidade corporal ou percentual de gordura corporal ${ }^{4}$. As equações se revelam exatas para indivíduos semelhantes em idade, sexo, estado de treinamento, adiposidade e raça em relação ao grupo na qual foram obtidas ${ }^{24}$. No entanto, a técnica de PC apresenta algumas limitações. Quando esses critérios são obedecidos, a gordura corporal predita para um indivíduo costuma oscilar entre 3 e 5\% do estimado a partir da $\mathrm{PH}^{4}$. À pessoa que realiza as mensurações das pregas cutâneas requerese acurácia e precisão na aplicação desta técnica. A calibração do compasso utilizado também pode contribuir para os erros de mensuração $0^{25}$. Além disso, uma equação elaborada por um pesquisador, que pode demonstrar alta validade para sua amostra, pode produzir grandes erros quando aplicada em grupos ou situações diferentes ${ }^{6}$

A IB pode ser considerada uma técnica rápida, não-invasiva e razoavelmente barata comparada com outros métodos como DXA e $\mathrm{PH}^{1}$. No entanto, Segal ${ }^{1}$ aponta dois problemas quanto a sua utilização no esporte: fatores fisiológicos alterados e limitações quanto à especificidade das equações de predição. Porém, a maior limitação da IB está na baixa sensibilidade às mudanças na composição corporal provocadas pelas sessões de treinamento e mudanças bruscas na dieta, tão frequentes na rotina dos atletas de nível competitivo ${ }^{4,7}$. Entretanto, estudos consideram que a IB poderá representar a técnica mais utilizada para estimar a composição corporal e a condição de hidratação em atletas em alguns anos ${ }^{11}$.

Deste modo, revisões críticas são importantes ferramentas, pois são capazes de sintetizar resultados a partir de avaliação criteriosa de um conjunto de estudos sobre determinado assunto e direcionar a atuação dos profissionais. Vale ressaltar que este tipo de estudo pode apresentar falhas, pois pode não abranger todas as vantagens e limitações de cada estudo.

Estudos futuros são necessários com o intuito de desenvolver e validar equações de estimativa da composição corporal específicas para diferentes idades, gênero e modalidade esportiva.

\section{CONCLUSÃO}

Analisando os estudos selecionados, pode-se concluir que a técnica de PC é mais confiável quando comparada com IB para estimar a composição corporal em atletas. $\mathrm{O}$ maior número e controle dos estudos, além da maior variedade de equações para as diferentes modalidades são os principais motivos da maior confiabilidade na PC. A grande interferência das mudanças bruscas no organismo do atleta em situações pós-treino parece ser a principal limitação da técnica de IB nessa população.

\section{REFERÊNCIAS BIBLIOGRÁFICAS}

1. Segal KR. Use of bioelectrical impedance analysis measurements as an evaluation for participating in sports. Am J Clin Nutr 1996;64(3 Suppl):469S-471S. 
2. De Lorenzo A, Bertini I, Iacopino L, Pagliato E, Testolin C, Testolin G. Body composition measurement in highly trained male athletes. A comparison of three methods. J Sports Med Phys Fitness 2000;40(2):178-183.

3. Lukaski HC, Johnson PE, Bolonchuk WW, Lykken GI . Assessment of fat-free mass using bioelectrical impedance measurements of the human body. Am J Clin Nutr 1985;41(4):810-817.

4. McArdle WD, Katch FI, Katch VL. Fisiologia do exercício: energia nutrição e desempenho humano. Rio de Janeiro: Guanabara Koogan; 2003.

5. Oppliger RA, Nielsen DH, Vance CG. Wrestlers' minimal weight: anthropometry, bioimpedance, and hydrostatic weighing compared. Med Sci Sports Exerc 1991;23(2):247-253.

6. Eliakim A, Ish-Shalom S, Giladi A, Falk B, Constantini $\mathrm{N}$. Assessment of body composition in ballett dancers: correlation among anthropometric measurements, bioelectrical impedance analysis, and dual-energy X-ray absorptiometry. Int J Sports Med 2000;21(8):598-601.

7. Yannakoulia M, Keramopoulos A, Tsakalakos N, Matalas AL. Body composition in dancers: the bioelectrical impedance method. Med Sci Sports Exerc 2000;32(1):228-234.

8. Huygens W, Claessens AL, Thomis M, Loos R, Van Langendonck L, Peeters M, et al. Body composition estimations by IB versus anthropometric equations in body builders and other power athletes. J Sports Med Phys Fitness 2002;42(1):45-55.

9. Andreoli A, Melchiorri G, Volpe SL, Sardella F, Iacopino L, De Lorenzo A. Multicompartment model to assess body composition in professional water polo players. J Sports Med Phys Fitness 2004;44(1):38-43.

10. Utter AC, Nieman DC, Mulford GJ, Tobin R, Schumm $\mathrm{S}$, McInnis T, et al. Evaluation of leg-to-leg BIA in assessing body composition of high-school wrestlers. Med Sci Sports Exerc 2005;37(8):1395-1400.

11. Ostojic SM. Estimation of body fat in athletes: skinfolds vs bioelectrical impedance. J Sports Med Phys Fitness 2006;46(3):442-446.

12. Webster BL, Barr SI. Body composition analysis of female adolescent athletes: comparing six regression equations. Med Sci Sports Exerc 1993;25(5):648-653.

13. Stewart AD, Hannan WJ. Prediction of fat and fat-free mass in male athletes using dual X-ray absorptiometry as the reference method. J Sports Sci 2000;8(4):263-274.

14. Hortobagyi T, Israel RG, Houmard JA, McCammon MR, O'Brien KF. Comparison of body composition assessment by hydrodensitometry, skinfolds, and multiple site near-infrared spectrophotometry. Eur J Clin Nutr 1992;46(3):205-211.

15. Saito K, Nakaji S, Umeda T, Shimoyama T, Sugawara $\mathrm{K}$, Yamamoto Y. Development of predictive equations for body density of sumo wrestlers using B-mode ultrasound for the determination of subcutaneous fat thickness. Br J Sports Med 2003;37(2):144-148.
16. Viitala P, Newhouse IJ. Vitamin E supplementation, exercise and lipid peroxidation in human participants. Eur J Appl Physiol 2004;93(1-2):108-115.

17. Utter AC, Scott JR, Oppliger RA, Visich PS, Goss FL, Marks BL, et al. A comparison of leg-to-leg bioelectrical impedance and skinfolds in assessing body fat in collegiate wrestlers. J Strength Cond Res 2001;15(2):157-160.

18. Hetzler RK, Kimura IF, Haines K, Labotz M, Smith J. A comparison of bioelectrical impedance and skinfold measurements in determining minimum wrestling weights in high school wrestlers. J Athl Train 2006;41(1):46-51.

19. Saunders MJ, Blevins JE, Broeder CE. Effects of hydration changes on bioelectrical impedance in endurance trained individuals. Med Sci Sports Exerc 1998;30(6):885-892.

20. Bland JM, Altman DG. Statistical methods for assessing agreement between two methods of clinical measurement. Lancet 1986;1(8476):307-310.

21. Lukaski HC, Bolonchuk WW, Siders WA, Hall CB. Body composition assessment of athletes using bioelectrical impedance measurements. J Sports Med Phys Fitness 1990;30(4):434-440.

22. Sun G, French CR, Martin GR, Younghusband B, Green RC, Xie YG, et al. Comparison of multifrequency bioelectrical impedance analysis with dual-energy X-ray absorptiometry for assessment of percentage body fat in a large, healthy population. Am J Clin Nutr 2005;81(1):74-78.

23. De Lorenzo A, Sorge SP, Iacopino L, Andreoli A, de Luca PP, Sasso GF. Fat-free mass by bioelectrical impedance vs dual-energy X-ray absorptiometry (DXA). Appl Radiat Isot 1998;49(5-6):739-741.

24. Nindl BC, Kraemer WJ, Emmert WH, Mazzetti SA, Gotshalk LA, Putukian M, et al. Comparison of body composition assessment among lean black and white male collegiate athletes. Med Sci Sports Exerc 1998;30(5):769-776.

25. Gore CJ, Carlyon RG, Franks SW, Woolford SM. Skinfold thickness varies directly with spring coefficient and inversely with jaw pressure. Med Sci Sports Exerc 2000;32(2):540-546.
Endereço para correspondência
Rafael Deminice
Universidade de São Paulo
Faculdade de Medicina de Ribeirão Preto
Avenida Bandeirantes, 3900
Monte Alegre
14049-900 - Ribeirão Preto, SP. Brasil
E-mail: deminice@ig.com.br 